\title{
Short-Range Structural Connections Are More Severely Damaged in Early-Stage MS
}

\author{
(D) H. Wu, (D) C. Sun, (D) X. Huang, (D) R. Wei, (D) Z. Li, (D) D. Ke, (D) Rai, and (D) H. Liang
}

\begin{abstract}
BACKGROUND AND PURPOSE: Long-range connections are more severely damaged and relevant for cognition in long-standing MS. However, the evolution of such coordinated network damage in patients with MS is unclear. We investigated whether short- and long-range structural connections sustained equal damage in early-stage MS.
\end{abstract}

MATERIALS AND METHODS: Sixteen patients with early-stage MS and 17 healthy controls were scanned by high-resolution, multishell diffusion imaging on 7T MR imaging and assessed cognitively. We investigated macrostructural properties in short- and longrange fibers and of microstructural metrics derived from 2 quantitative diffusion MR imaging models: DTI and neurite orientation dispersion and density imaging.

RESULTS: Patients had significant WM integrity damage - that is, higher radial diffusivity and a lower intracellular volume fraction in the focal WM lesions. Compared with the healthy controls, the patients had noticeable microstructure changes in both short- and long-range fibers, including increased radial diffusivity, mean diffusivity, and axial diffusivity. Z scores further indicated greater damage in the short-range fibers than in the long-range fibers.

CONCLUSIONS: Our findings demonstrate that more severe demyelination preceding axonal degeneration occurs in short-range connections but not in long-range connections in early-stage MS, suggesting the possibility that there are cortical lesions that are undetectable by current MR imaging.

ABBREVIATIONS: $A D=$ axial diffusivity; EDSS = Expanded Disability Status Scale; FA = fractional anisotropy; $f_{\text {icvf }}=$ intracellular volume fraction; $\mathrm{HC}=$ healthy control; $\mathrm{MD}=$ mean diffusivity; $\mathrm{NODDI}=$ neurite orientation dispersion and density imaging; $\mathrm{ODI}=$ Orientation Dispersion Index; RD $=$ radial diffusivity

M $S$ has been histologically characterized by the aggregation of inflammation, demyelination, gliosis, and axonal transection that ultimately leads to neurologic disability in young

Received August 20, 2021; accepted after revision December 11.

From the Department of Neurology (H.W., X.H., R.W., D.K., H.L.), First Affiliated Hospital, Key Laboratory of Biomedical Engineering of Ministry of Education (C.S., Z.L.), College of Biomedical Engineering and Instrument Science, and Department of Physical Medicine and Rehabilitation of the Affiliated Sir Run Run Shaw Hospital and Interdisciplinary Institute of Neuroscience and Technology (R.B.), School of Medicine, Zhejiang University, Hangzhou, China.

This work was supported by the National Natural Science Foundation of China (NSFC) (Grant No. 81873894) and the Natural Science Foundation of Zhejiang Province, China (Grant No. LR20H180001), China middle-young VG Clinical Research Fund (2017-CCA-VG-047), National key research and development plan of China (2019YFC0118203, 2016YFC1306402), Zhejiang key research and development plan (2017C03011).

H. Liang is senior author. H. Liang and R. Bai contributed equally to this work. Please address correspondence to Ruiliang Bai, PhD, 268 Kaixuan Rd, South Central Building, Room 708, Hangzhou, Zhejiang, China 310027; e-mail:

ruiliangbai@zju.edu.cn

- Indicates open access to non-subscribers at www.ajnr.org

Indicates article with online supplemental data.

http://dx.doi.org/10.3174/ajnr.A7425 adults. MS can present with highly variable symptoms such as loss of vision, limb weakness, sensory loss, ataxia, and cognitive impairment. ${ }^{1,2}$ Neuroimaging studies have helped to define the pathologic substrates and microstructure (eg, demyelination) alternations of this disorder in focal lesions and diffusely abnormal tissue. ${ }^{3,4}$ While the specific WM fiber changes remain elusive, these local abnormalities in tissue microstructure are not enough to explain the clinically observed functional impairment. ${ }^{5,6}$ Nevertheless, there is growing evidence that neurologic impairment in MS is determined more by specific large-scale functional network alterations than by local tissue microstructural alterations. ${ }^{7,8}$

The subtle balance between short- and long-range connections contributes to the integration and segregation of the brain connectome. Connections of different lengths are found in different locations across the brain; short-range fibers are more abundant than their long-range counterparts, and the short-range fibers connect adjacent anatomic gyri that integrate multimodal information. Concurrently, long-range connections allow rapid 
and efficient interareal communication (eg, among cortical regions where tracts may cross the centrum semiovale) or diversification of information. ${ }^{9-12}$ Cognitive impairment is one of the primary symptoms of MS, occurring in up to $70 \%$ of patients. ${ }^{2}$ The specific damage to long-range connections in patients with long-standing MS would affect cognition by reducing the global efficiency of brain networks. ${ }^{13}$ However, it is still unclear when such distinct connectivity adjustments happen and whether this is a unique feature of patients with long-standing MS. In-depth knowledge of the evolution of the balance between short- and long-range connections during disease progression would significantly increase the understanding of the clinical symptoms of MS.

Previous studies have found that cortical demyelination occurs early, is common in patients with MS, and would affect the U-fibers more. ${ }^{4,14}$ Thus, we propose the following hypothesis: Short-range connections might be more severely damaged than long-range connections in early-stage MS, which is different from long-lasting MS. To make a definite determination, we explored the potential damage of long- and short-range structural connections in patients with early-stage MS who exhibited significant cognitive decline in this study. High-resolution DWI with multishell acquisition and structural MR imaging was performed on 16 patients with early-stage MS and 17 healthy controls (HCs), to quantify the damage to shortand long-range connections.

\section{MATERIALS AND METHODS Participants}

For this prospective study, we recruited 16 patients with earlystage relapsing-remitting MS with a disease duration of $<10$ years. ${ }^{15,16}$ Patients had been diagnosed with MS according to the 2017 revisions of the McDonald criteria, ${ }^{17}$ had been free of clinical relapse, and had not required steroid treatment for at least 1 month before participation. Exclusion criteria were evidence of other structural brain diseases; a history of neuropsychiatric disorders, cognitive impairment, or other significant medical conditions; or contraindications to MR imaging. In addition, 17 ageand education level-matched HCs were recruited. Informed consent was obtained from each participant in this study, and the protocol was approved by the First Affiliated Hospital, School of Medicine, Zhejiang University. The cohorts have not been described in any previous publication.

\section{Clinical Assessment}

Neuropsychological tests were used to assess the most frequently impaired cognitive domains in MS. The tests included the Symbol Digit Modalities Test ${ }^{18}$ for information-processing speed, the Verbal Fluency Test ${ }^{19}$ for executive function, the Digit Span Test $^{8}$ for attention, and the Auditory Verbal Learning Test ${ }^{20}$ for episodic memory. Also, the Mini-Mental State Examination and the Montreal Cognitive Assessment ${ }^{21}$ were performed to assess global cognitive function, and the Expanded Disability Status Score (EDSS) ${ }^{22}$ was performed for overall disability. The mean of the neuropsychological assessment of the patients was further analyzed by evaluating the deviation from the mean values based on the HC group. The results of each patient's neuropsychological tests were regarded as normal if they declined $<1.5$ SDs from the mean values of the HC group; ${ }^{23}$ patients were diagnosed as cognitively impaired when the results of at least 2 tests had declined. $^{24}$

\section{Image Acquisition}

All MR imaging data were acquired using a 7T MR imaging scanner (Magnetom; Siemens) with a 32-channel Nova Medical Head Coil (Siemens). For the structural MR imaging scans, T1weighted images were acquired using a 3D MP2RAGE sequence with $0.8-\mathrm{mm}$ isotropic resolution. The $3 \mathrm{D}$ sampling perfection with application-optimized contrasts by using different flip angle evolution (SPACE sequence; Siemens) T2 sequence was performed with a voxel size of $0.8 \times 0.8 \times 0.8 \mathrm{~mm}^{3}$. A multiband $2 \mathrm{D}$ EPI sequence was used for the multishell DWI protocol: $1.5-\mathrm{mm}$ isotropic resolution, 3 shells $\left(1000 / 2000 / 3000 \mathrm{~s} / \mathrm{mm}^{2}, 40\right.$ directions for each shell, single repetitions), and 8 repetitions without diffusion weighting; $b=0 \mathrm{~s} / \mathrm{mm}^{2}$. The gradient directions were optimized to maximize the minimal angle between directions and cross shells using the DMRITool ${ }^{25}$ (https://diffusionmritool. github.io).

\section{DWI Data Preprocessing and Modeling}

The image-processing pipeline is detailed below and summarized in Fig 1. All the preprocessing steps for raw DWIs, including eddy currents, motion, and EPI geometric distortion correction, were processed in the TORTOISE (Version 3.2; https://tortoise. nibib.nih.gov/) software package, ${ }^{26}$ and a T2-weighted image was used as the reference image. The output images with opposite phase-encoding were then used for EPI geometric distortion correction using the DR-BUDDI toolbox. ${ }^{27}$ The preprocessed diffusion data sets were then used for further diffusion metrics calculations.

The DTI reconstruction was performed in TORTOISE using $b=0$ and $1000 \mathrm{~s} / \mathrm{mm}^{2}$, along with the calculation of fractional anisotropy (FA), axial diffusivity ( $\mathrm{AD})$, radial diffusivity $(\mathrm{RD})$, and mean diffusivity (MD). The neurite orientation dispersion and density imaging (NODDI) reconstruction using the 3-shell data was performed in the NODDI toolbox in Matlab (Version 1.01; University College London, London, United Kingdom, http://mig.cs.ucl.ac.uk/ index.php?n=Tutorial.NODDImatlab). ${ }^{28}$ Maps of NODDI metrics, including intracellular volume fraction $\left(f_{\text {icve }}\right)$ and the Orientation Dispersion Index (ODI) were then generated.

\section{WM Lesion ROI Identification and Analysis}

The individual T1-weighted image was linearly registered to each patient's $B_{0}$ image. A whole-brain WM mask was segmented with coregistered T1-weighted images. The ROIs of patients with WM lesions were manually drawn on T1-weighted images with the complementing guidance of the T2-weighted images. The lesion load of each type of fiber was further defined as the fraction of fiber volume overlapped with the WM lesions (ie, volume of the fiber overlapped with WM lesions divided by the whole volume of this type of fiber).

$\mathrm{B}_{0}$ images of each $\mathrm{HC}$ were first linearly (FMRIB Linear Image Registration Tool [FLIRT]; http://www.fmrib.ox.ac.uk//fsl/ fslwiki/FLIRT) and then nonlinearly registered to the same diffusion-weighted images in each patient with MS to obtain the 


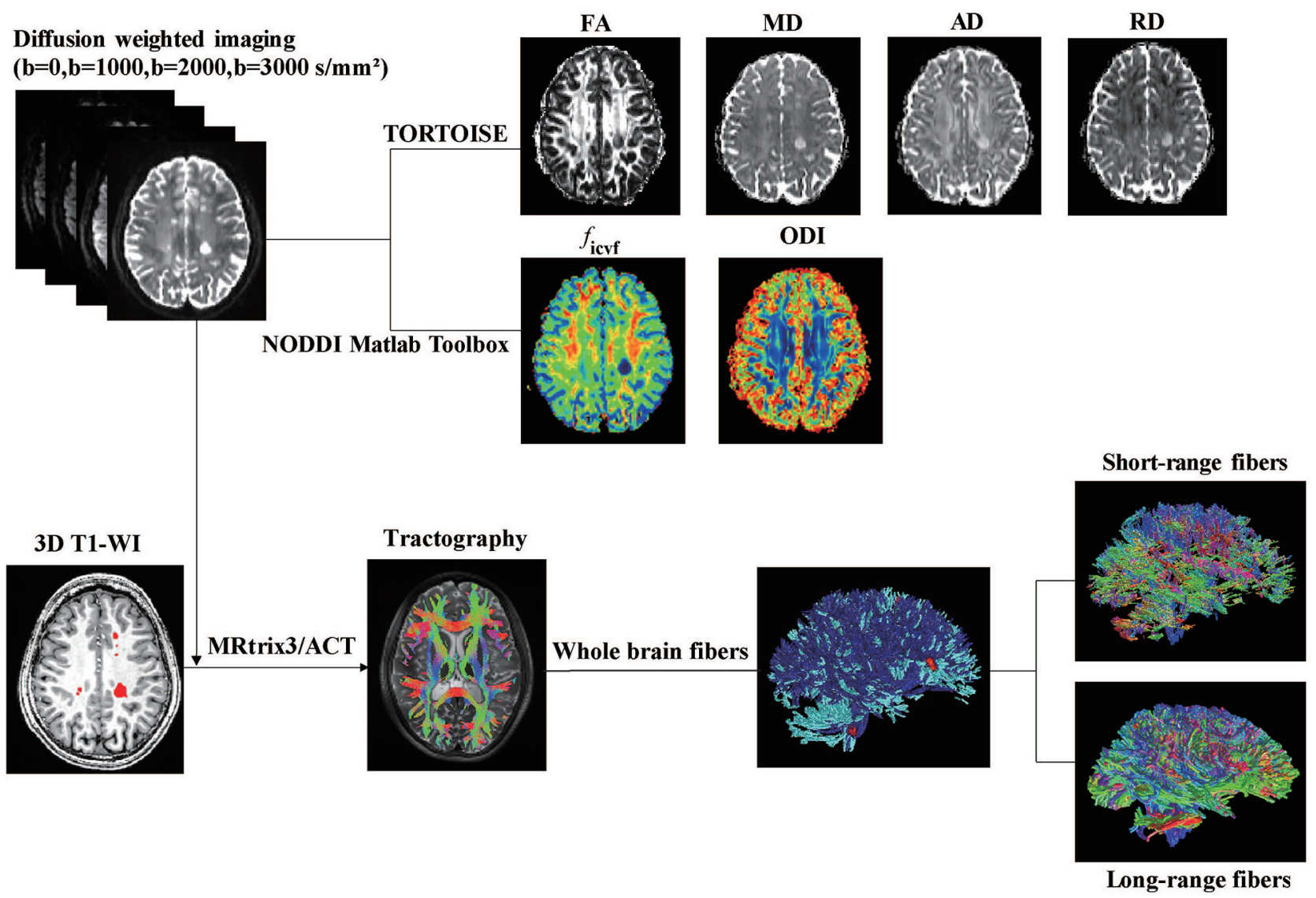

FIG 1. Overview of the image acquisition and processing pipeline.

location-matched ROIs in the HC group. Finally, the mean value of the diffusion MR imaging metrics for each ROI or whole WM was calculated.

\section{Tractography}

The probabilistic tractography procedure was performed on the MRtrix3 software package (http://www.mrtrix.org). ${ }^{29}$ Five tissuetype segmented images obtained from the T1-weighted images were used for the Anatomically-Constrained Tractography framework. ${ }^{30}$ We estimated the response functions and computed the fiber orientation distributions from the preprocessed DWI. Next, streamline tractography was performed by randomly seeding 20 million streamlines with default settings filtered to 2 million streamlines using spherical-deconvolution informed filtering of tractograms. $^{31}$

We segmented structural fiber tracts into short- and longrange fibers as previously described. ${ }^{13}$ Then, we calculated the number and average length of the fibers in the whole WM. In addition, the mean of the microstructure metrics (eg, FA, $f_{\text {icvf }}$ ) along the selected fiber tracts was calculated. We used the $z$ score to compare the MR imaging metrics between short- and longrange fibers on a common scale. The formula for calculating the $z$ score is $z=(x-\mu) / \sigma$, where $x$ is the mean value of the MR imaging metrics of short or long fibers in the MS group, $\mu$ is the mean value of corresponding MR imaging metrics in the MS group, and $\sigma$ is the SD of the corresponding MR imaging metrics in the HC group. ${ }^{13}$

\section{Statistical Analysis}

Statistical analysis was performed using SPSS software (Version 24; IBM). Group differences in unpaired parametric data were assessed using the independent-samples $t$ test for continuous variables and the $\chi^{2}$ test for categoric variables. The results of the $z$ scores of short- and long-range fibers were analyzed using the 1sample $t$ test on a convergence of the average value to zero to compare with the HCs. The paired analysis of short- and longrange homologous fibers for each patient with MS was compared with the paired $t$ tests. For patients with MS, intersubject bivariate correlation was performed between the MR imaging metrics and the clinical characteristics, while age and education level were treated as covariates of no interest. The level of statistical significance after false discovery rate correction was set at $P<.05$ for all statistical analyses.

\section{RESULTS}

\section{Disability, Cognition, and Brain Volume}

Demographic, clinical, and MR imaging characteristics for the patients with MS and the HCs are presented in Tables 1 and 2. In the MS cohort, patients had severe damage in several cognitive domains (Table 2). A total of 10 of 16 patients (62.5\%) were classified as cognitively impaired. Among the remaining 6 patients, 3 (18.8\%) showed isolated cognitive impairment in the form of reduced information-processing speed. Further brain volume comparison did not detect a significant difference in WM volume. 
Table 1: Demographic, clinical, and MR imaging volumetric characteristics of all participants ${ }^{\mathrm{a}}$

\begin{tabular}{|c|c|c|c|}
\hline & MS $(n=16)$ & $\mathrm{HC}(n=17)$ & $P$ Value \\
\hline \multicolumn{4}{|l|}{ Demographics } \\
\hline Age (yr) & 31.94 (SD, 9.72) & 28.04 (SD, 4.51) & .063 \\
\hline Sex (male/female) & $8 / 8$ & $8 / 9$ & .866 \\
\hline Education (yr) & 13.81 (SD, 3.17) & $16.00(\mathrm{SD}, 3.82)$ & .084 \\
\hline \multicolumn{4}{|l|}{ Clinical data } \\
\hline $\operatorname{DMDs}(\%)^{\mathrm{b}}$ & $62.5 \%(10 / 16)$ & NA & NA \\
\hline EDSS score & $1.0(0.0-5.0)$ & NA & NA \\
\hline Disease duration (yr) & $3.17(0.36-9.42)$ & NA & NA \\
\hline \multicolumn{4}{|l|}{ MR imaging volumetric data } \\
\hline Whole-brain volume (L) & $1.51(S D, 0.16)$ & $1.54(S D, 0.24)$ & .647 \\
\hline Brain GM volume $(\mathrm{L})$ & 0.81 (SD, 0.13) & $0.87(S D, 0.13)$ & .477 \\
\hline Brain WM volume (L) & 0.60 (SD, 0.11$)$ & $0.63(S D, 0.10)$ & .202 \\
\hline Lesion volume $(\mathrm{mL})$ & $4.09(0.20-14.19)$ & NA & NA \\
\hline
\end{tabular}

Note:-DMDs indicates disease-modifying drugs; NA, not applicable.

${ }^{a}$ All continuous data are displayed as mean (SD), except the score, disease duration, and lesion volume, which are shown as median (range).

${ }^{b}$ Indicates treatment with disease-modifying drugs; $37.5 \%$ (6/16) of patients were treated with teriflunomide; and 25\% (4/16), with an anti-CD20 monoclonal antibody (rituximab).

Table 2: Summary of cognitive measures ${ }^{\mathrm{a}}$

\begin{tabular}{|c|c|c|c|}
\hline NP Test & MS & $\mathrm{HC}$ & $\begin{array}{c}P \\
\text { Value }\end{array}$ \\
\hline \multicolumn{4}{|l|}{$\begin{array}{l}\text { Global cognitive } \\
\text { function }\end{array}$} \\
\hline MMSE & 27.73 (SD, 2.94) & 29.69 (SD, 0.60) & .023 \\
\hline MoCA & 25.00 (SD, 5.01) & $27.88(S D, 1.71)$ & .051 \\
\hline \multicolumn{4}{|l|}{ Processing speed } \\
\hline SDMT & 36.89 (SD, 15.79) & 56.85 (SD, 8.66) & $<.001$ \\
\hline \multicolumn{4}{|l|}{$\begin{array}{l}\text { Verbal episode } \\
\text { memory }\end{array}$} \\
\hline AVLT-SFR & 7.00 (SD, 3.67) & 10.06 (SD, 2.93) & .019 \\
\hline AVLT-LFR & 7.69 (SD, 4.57) & 10.25 (SD, 2.79) & .074 \\
\hline AVLT-DI & $9.92(\mathrm{SD}, 3.59)$ & 13.00 (SD, 2.33) & .011 \\
\hline \multicolumn{4}{|c|}{ Executive function } \\
\hline VFT & 33.67 (SD, 9.85) & $42.12(\mathrm{SD}, 8.31)$ & .013 \\
\hline \multicolumn{4}{|l|}{ Attention } \\
\hline DST & 13.73 (SD, 2.24) & $16.24(S D, 1.68)$ & .001 \\
\hline
\end{tabular}

Note:-MMSE indicates Mini-Mental State Examination; MoCA, Montreal Cognitive Assessment; SDMT, Symbol Digit Modalities Test; AVLT, auditory verbal learning test; SFR, short-delayed free recall; LFR, long-delayed free recall; DI, discrimination index; VFT, verbal fluency test; DST, Digit Span Test; NP test, neuropsychological test.

${ }^{a}$ Data are mean (SD).

\section{Microstructure Metrics in Focal and Diffuse Lesions}

The statistical analysis of the microstructure metrics assessed with the DWI of the WM lesion ROIs and whole WM is shown in Table 3. All quantitative metrics, except for ODI, of the focal lesion ROIs had a significant difference between the MS and HC groups. As for the whole WM, no significant differences were found between these 2 groups.

\section{Difference of the Whole-Brain Fibers between MS and HC Cohorts}

There were no significant differences between the MS and HC cohorts in the overall number or average length of whole-brain fibers (Table 3). However, when short- and long-range fibers were observed independently, further analysis of the microstructure metrics of the short- and long-range fibers between the HC and MS cohorts revealed that the short-range fibers in patients with MS had a significant change in FA $(P<.05), \mathrm{MD}, \mathrm{AD}$, and $\mathrm{RD}$ (Fig 2). Long-range fibers in patients with MS showed a significant increase in $\mathrm{MD}, \mathrm{AD}$, and $\mathrm{RD}$.

\section{Direct Comparison of Damage (Z Scores) to Short- and Long-Range Connections}

The WM lesion ROIs overlapped with 6.66\% (range, 1.87\%9.55\%) of the whole-brain fibers in patients with MS. The $z$ score analysis of fiber properties is presented in Fig 3, in which both short- and long-range fibers show a significant increase in MD, $\mathrm{AD}$, and $\mathrm{RD}$ in patients with MS compared with HCs. The increased $\mathrm{MD}, \mathrm{AD}$, and $\mathrm{RD}$ indicate the potential damage of WM integrity along short- and long-range fibers (Fig 3).

Further comparisons between the $z$ scores of short- and longrange fibers show that the short-range connections have a larger increase than the long-range connections in both $\mathrm{MD}$ and $\mathrm{RD}$. The MD $z$ score was 2.57 (SD, 2.78) (short) versus 1.06 (SD, 1.05) (long), $P<.05$; and the RD $z$ score was 3.10 (SD, 3.44) (short) versus 0.75 ( $\mathrm{SD}, 1.29$ ) (long), $P<.01$. The other microstructure metrics also showed the same tendency: The short-range fibers were more severely damaged than the long-range fibers.

\section{Associations with Clinical Variables}

No significant associations were found between MR imaging metrics and clinical variables after adjustment was made for the effect of age and years of education.

\section{DISCUSSION}

Long-range structural connections are more severely damaged in patients with long-standing MS. ${ }^{13}$ We have observed that the short-range connections are more severely damaged in earlystage MS, suggesting that the structural connections are altered differently during the progress of MS. In this study, the members of the MS cohort were in the early stages of disability, with a median EDSS score of 1.0, and had distinct cognitive impairment without obvious brain atrophy.

Microstructure Properties in MS WM Lesions and Whole WM Our results show that in subjects with MS, WM lesion ROIs have higher $\mathrm{MD}, \mathrm{AD}$, and $\mathrm{RD}$ and lower $\mathrm{FA}$ and $f_{\text {icvf }}$ values compared with HCs. These results suggest there is damage to the WM integrity in these focal lesion areas, which is consistent with previous findings. ${ }^{32,33}$ However, there were no significant differences in the microstructure metrics of whole $\mathrm{WM}$ as found in previous 


\begin{tabular}{|c|c|c|c|c|c|c|}
\hline & \multicolumn{3}{|c|}{ WM Lesion ROls } & \multicolumn{3}{|c|}{ Whole-Brain WM } \\
\hline & MS & $\mathrm{HC}$ & $P$ Value ${ }^{b}$ & MS & $\mathrm{HC}$ & $P$ Value $^{\mathrm{b}}$ \\
\hline \multicolumn{7}{|l|}{ DTI } \\
\hline FA & 0.35 (SD, 0.06$)$ & 0.41 (SD, 0.06) & .010 & 0.39 (SD, 0.02) & 0.41 (SD, 0.01) & .655 \\
\hline$M D$ & $0.92(S D, 0.17)$ & $0.74(S D, 0.04)$ & $<.001$ & $0.76(S D, 0.06)$ & 0.74 (SD, 0.03$)$ & .334 \\
\hline$A D$ & 1.28 (SD, 0.21) & 1.08 (SD, 0.04) & .002 & $1.28(S D, 0.03)$ & 1.07 (SD, 0.03) & .327 \\
\hline $\mathrm{RD}$ & 0.77 (SD, 0.15) & 0.57 (SD, 0.06) & $<.001$ & 0.59 (SD, 0.03) & $0.56(S D, 0.02)$ & .555 \\
\hline \multicolumn{7}{|l|}{ NODDI } \\
\hline$f_{\text {icvf }}$ & 0.55 (SD, 0.12) & 0.69 (SD, 0.05) & .001 & 0.69 (SD, 0.06) & 0.71 (SD, 0.05) & .914 \\
\hline ODI & 0.30 (SD, 0.07) & $0.34(S D, 0.05)$ & .109 & 0.34 (SD, 0.02$)$ & $0.33(S D, 0.02)$ & .334 \\
\hline \multicolumn{7}{|l|}{ Fiber } \\
\hline No. & $6308(1319,11,641)$ & $7582(1204,13,494)$ & .780 & 199,230 (SD, 46,819) & 208,260 (SD, 60,599) & .905 \\
\hline Length & 35.11 (SD, 12.21) & $39.73(\mathrm{SD}, 8.32)$ & .211 & $22.39(\mathrm{SD}, 6.24)$ & 22.73 (SD, 7.21) & .846 \\
\hline
\end{tabular}

${ }^{a}$ Data are mean (SD) except for the number of fibers through lesion areas, which are median (range).

${ }^{b}$ False discovery rate-corrected $P$ values.
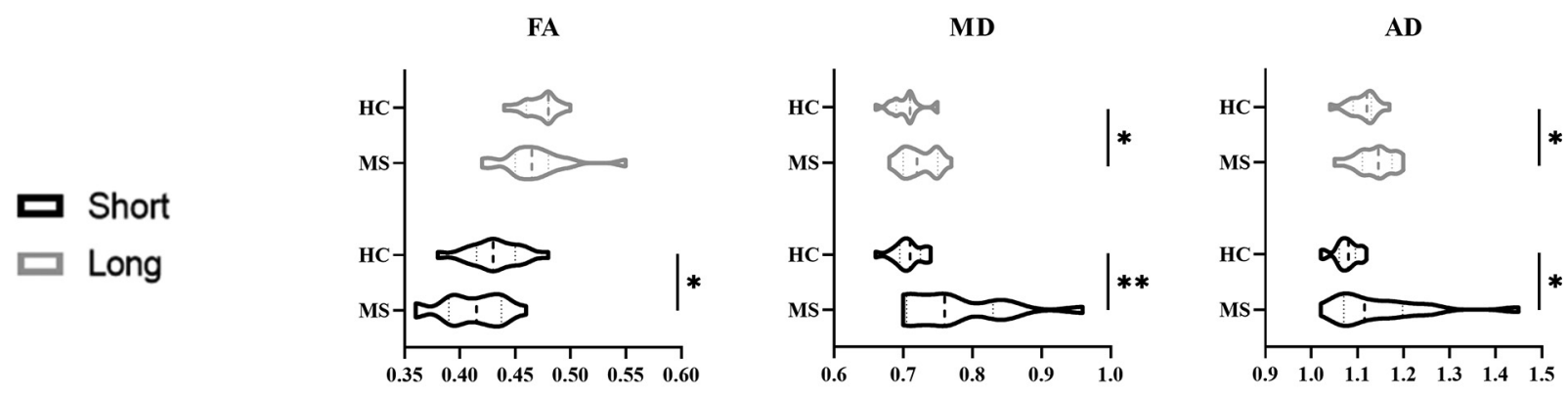

Length of fibers $(\log 2)$

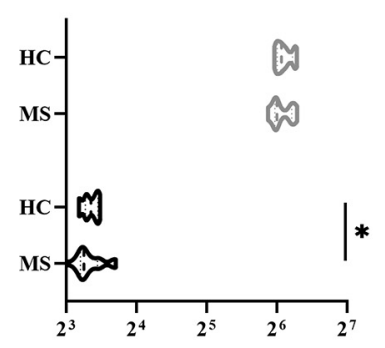

RD

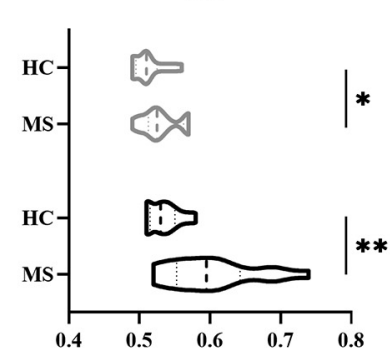

$f_{\text {icvf }}$

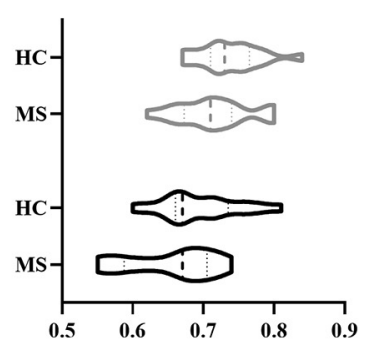

ODI

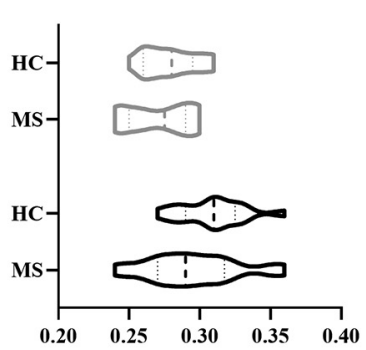

FIG 2. The fiber microstructure metrics of whole-brain short- (black) and long-range (gray) connections in patients with MS and HCs. log2 indicates the length of fibers was denoted in a logarithmic manner with base 2.

studies, ${ }^{32,34}$ even though these changes have been observed in patients who have had MS for a longer time ( $>10$ years). ${ }^{13,33}$ This finding is not surprising because the volume of the detectable WM lesions is relatively small ( $\sim 4 \mathrm{~mL}$ on average) and takes only a small portion of the whole WM in early-stage MS, while the WM lesions are much larger in long-lasting MS (eg, $\sim 670 \mathrm{~mL}^{13}$ ). In addition, previous studies have found that there was no significant difference in diffusion metrics in the normal-appearing WM of subjects with early-stage MS compared with controls. ${ }^{32,34}$ These 2 factors could make the changes of the whole-WM averaged diffusion MR imaging metrics relatively small, which means a large sample size is needed to make such changes significant in statistics.

\section{Short-Range Connections Are More Severely Damaged in Early-Stage MS}

In this study, the short-range fibers showed a more severe increase in the mean $\mathrm{RD}$ and $\mathrm{MD}$ values than the long-range fibers, a finding different from the results in patients with longstanding MS. ${ }^{13}$ Previous studies have shown that notable changes in $\mathrm{RD}$ are considered as noninvasive surrogate markers for myelin integrity, and myelin integrity may influence the diffusivity along the perpendicular direction of fibers; $\mathrm{AD}$ and $f_{\text {icvf }}$ correspond more to axonal loss. ${ }^{28,35}$

These results agree with MS being primarily a demyelinating disease that ultimately results in axonal degeneration, and they are in line with reported pathologic findings and current dogma. ${ }^{36,37}$ The early damage to the shortrange fibers reflects the possibility that cortical lesions precede the degeneration of long-range fibers, as discussed below. Previous studies have demonstrated that short-range fibers have more myelin than long-range fibers. ${ }^{9,38}$ Injury to long axons in MS, which eventually causes the fragmentation of fibers, is secondary to myelin loss and may be the consequence of trophic disturbances after mixed outcomes 
FA
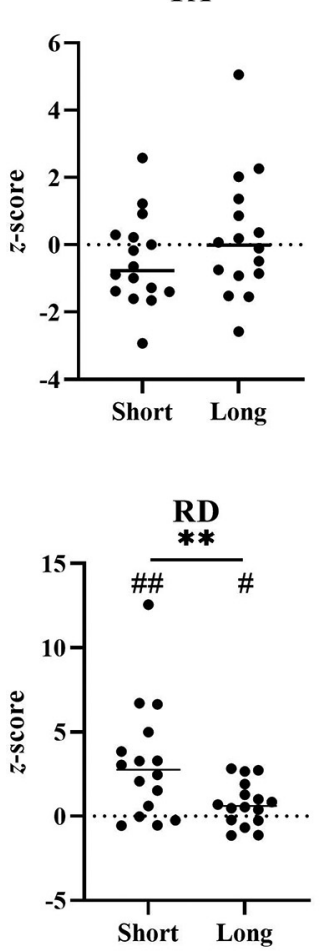
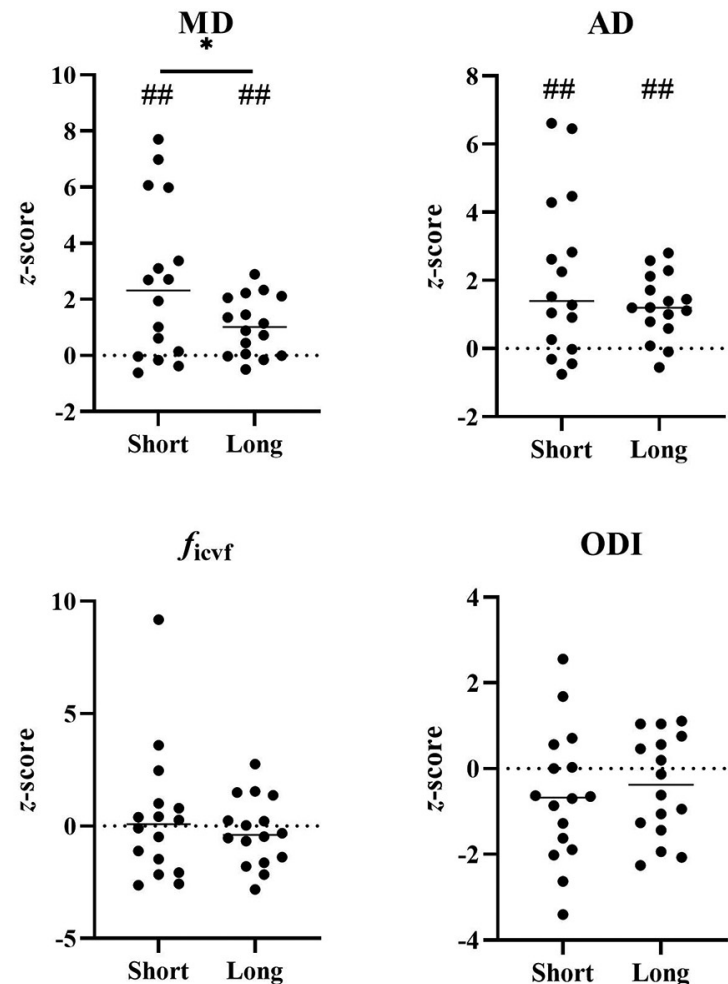

ODI

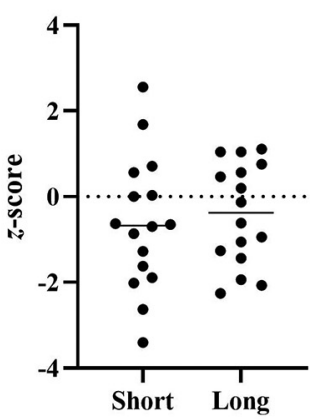

FIG 3. Severity of lesion damage ( $z$ score) compared between short- and long-range connections. The * represents a significant difference between short-and long-range fibers $(P<0.05)$; ** represents $P<0.01$. \# represents $P<0.05$ using the one sample $t$ test on a convergence of the average value to zero to compare with $\mathrm{HCs}$. \#\# represents $P<0.01$, FDR-corrected.

of cumulative interactions among oligodendrocytes, microglia, and neurons. ${ }^{36}$ Most interesting, the ODIs of short fibers showed no significant difference between 2 groups because ODI reflects only neurite orientation, which is not sensitive to demyelination. ${ }^{39}$

\section{Potential Invisible Leukocortical Lesions}

We found that the lesion load is higher for long-range connections, suggesting that WM lesions visible in T1-/T2-weighted MR imaging are located in the long-range fibers (Online Supplemental Data). This result seems to contradict the finding of more severe microstructural damage to the short-range connections compared with the long-range connections. However, it could also be that we have failed to identify some tissue injuries within the current T1-/T2-weighted MR imaging, especially those leukocortical lesions located in the connections between anatomic neighboring areas of the cerebral cortex (short-range fibers). ${ }^{11,38,40}$ These leukocortical lesions running through the Ufibers in patients have been defined as cortical type I lesions. ${ }^{41}$ Cortical demyelination occurs early and is common in patients with MS, and it develops independent from WM lesions. ${ }^{4,14}$ With the help of ultra-high-field MR imaging, some cortical pathology can be detected in patients with MS. ${ }^{42,43}$ Fortunately, we further confirmed by MP2RAGE that the U-fiber lesions appear in almost all patients with MS (12/16); this use of MP2RAGE provides a more precise anatomic delineation of white and gray matter pathology (Online Supplemental Data). ${ }^{43}$ However, it is still challenging to detect most cortical lesions due to their relatively small size, sparse inflammatory cell infiltration, and limited damage to the blood-brain barrier. All of these factors could result in invisible cortical lesions under current MR imaging methods. ${ }^{42}$ The more severe demyelination to the short-range connections found in this study suggests that fiber tract-based microstructure analysis might be another efficient way to see the invisible cortical lesions.

\section{Limitations and Future Work}

This study has some limitations. First, our sample of patients with early-stage MS was small and recruited cross-sectionally, but large and longitudinal studies are still needed to further validate the current study and monitor how the disease progresses. Second, our understanding of the relationship between short- and long-range connections is relatively immature, and future studies combining structural data with more functional data are needed to verify the intrinsic functional architecture of brain networks.

Most important, although we have carefully compared our results with the available and published pathology and radiology results and NODDI is a well-validated method, a direct comparison between MR imaging findings and the pathology of the same subject should be conducted to further confirm our interpretation.

\section{CONCLUSIONS}

We evaluated the damage to structural connections in patients with early-stage MS with cognitive impairment and found that short-range fibers are more vulnerable to damage in early-stage MS, indicating that cortical demyelination might precede the axonal loss of long-range fibers in patients with MS.

Disclosure forms provided by the authors are available with the full text and PDF of this article at www.ajnr.org.

\section{REFERENCES}

1. Reich DS, Lucchinetti CF, Calabresi PA. Multiple sclerosis. $N$ Engl J Med 2018;378:169-80 CrossRef Medline

2. Rocca MA, Amato MP, De Stefano N, et al; MAGNIMS Study Group. Clinical and imaging assessment of cognitive dysfunction in multiple sclerosis. Lancet Neurol 2015;14:302-17 CrossRef Medline

3. Filippi M, Rocca MA, Barkhof F, et al; Attendees of the Correlation between Pathological MRI Findings in MS workshop. Association between pathological and MRI findings in multiple sclerosis. Lancet Neurol 2012;11:349-60 CrossRef Medline

4. Kutzelnigg A, Lucchinetti CF, Stadelmann C, et al. Cortical demyelination and diffuse white matter injury in multiple sclerosis. Brain 2005;128:2705-12 CrossRef Medline 
5. Barkhof $\mathrm{F}$. The clinico-radiological paradox in multiple sclerosis revisited. Curr Opin Neurol 2002;15:239-45 CrossRef Medline

6. Mesaros S, Rocca MA, Riccitelli G, et al. Corpus callosum damage and cognitive dysfunction in benign MS. Hum Brain Mapp 2009;30:265666 CrossRef Medline

7. Cercignani M, Gandini Wheeler-Kingshott C. From micro- to macro-structures in multiple sclerosis: what is the added value of diffusion imaging. NMR Biomed 2019;32:e3888 CrossRef Medline

8. Dineen RA, Vilisaar J, Hlinka J, et al. Disconnection as a mechanism for cognitive dysfunction in multiple sclerosis. Brain 2009;132:23949 CrossRef Medline

9. Bajada CJ, Schreiber J, Caspers S. Fiber length profiling: a novel approach to structural brain organization. Neuroimage 2019;186:16473 CrossRef Medline

10. Mesulam MM. Large-scale neurocognitive networks and distributed processing for attention, language, and memory. Ann Neurol 1990;28:597-613 CrossRef Medline

11. Ouyang M, Kang H, Detre JA, et al. Short-range connections in the developmental connectome during typical and atypical brain maturation. Neurosci Biobehav Rev 2017;83:109-22 CrossRef Medline

12. Sepulcre J, Liu H, Talukdar T, et al. The organization of local and distant functional connectivity in the human brain. PLoS Comput Biol 2010;6:e1000808 CrossRef Medline

13. Meijer KA, Steenwijk MD, Douw L, et al. Long-range connections are more severely damaged and relevant for cognition in multiple sclerosis. Brain 2020;143:150-60 CrossRef Medline

14. Calabrese M, Gallo P. Magnetic resonance evidence of cortical onset of multiple sclerosis. Mult Scler 2009;15:933-41 CrossRef Medline

15. Engl C, Tiemann L, Grahl S, et al. Cognitive impairment in early MS: contribution of white matter lesions, deep grey matter atrophy, and cortical atrophy. J Neurol 2020;267:2307-18 CrossRef Medline

16. Portaccio E, Amato MP, Bartolozzi ML, et al. Neocortical volume decrease in relapsing-remitting multiple sclerosis with mild cognitive impairment. J Neurol Sci 2006;245:195-99 CrossRef Medline

17. Thompson AJ, Banwell BL, Barkhof F, et al. Diagnosis of multiple sclerosis: 2017 revisions of the McDonald criteria. Lancet Neurol 2018;17:162-73 CrossRef Medline

18. Langdon DW, Amato MP, Boringa J, et al. Recommendations for a Brief International Cognitive Assessment for Multiple Sclerosis (BICAMS). Mult Scler 2012;18:891-98 CrossRef Medline

19. Benedict RH, Amato MP, Boringa J, et al. Brief International Cognitive Assessment for MS (BICAMS): international standards for validation. BMC Neurol 2012;12:55 CrossRef Medline

20. Fink F, Eling P, Rischkau E, et al. The association between California Verbal Learning Test performance and fibre impairment in multiple sclerosis: evidence from diffusion tensor imaging. Mult Scler 2010;16:332-41 CrossRef Medline

21. Freitas S, Batista S, Afonso AC, et al. The Montreal Cognitive Assessment (MoCA) as a screening test for cognitive dysfunction in multiple sclerosis. Appl Neuropsychol Adult 2018;25:57-70 CrossRef Medline

22. Kurtzke JF. Rating neurologic impairment in multiple sclerosis: an Expanded Disability Status Scale (EDSS). Neurology 1983;33:144452 CrossRef Medline

23. Gromisch ES, Dhari Z. Identifying early neuropsychological indicators of cognitive involvement in multiple sclerosis. Neuropsychiatr Dis Treat 2021;17:323-37 CrossRef Medline

24. Nelson F, Datta S, Garcia N, et al. Intracortical lesions by $3 \mathrm{~T}$ magnetic resonance imaging and correlation with cognitive impairment in multiple sclerosis. Mult Scler 2011;17:1122-29 CrossRef Medline
25. Cheng J, Shen D, Yap PT, et al. Single- and multiple-shell uniform sampling schemes for diffusion MRI using spherical codes. IEEE Trans Med Imaging 2018;37:185-99 CrossRef Medline

26. Pierpaoli C, Walker L, Irfanoglu MO, et al. TORTOISE an integrated software package for processing of diffusion MRI data. In: Proceedings of the Annual Meeting of the International Society for Magnetic Resonance Medicine, Stockholm, Sweden. May 1-7, 2010

27. Irfanoglu MO, Modi P, Nayak A, et al. DR-BUDDI (Diffeomorphic Registration For Blip-Up Blip-Down Diffusion Imaging) method for correcting echo planar imaging distortions. Neuroimage 2015;106:28499 CrossRef Medline

28. Zhang H, Schneider T, Wheeler-Kingshott CA, et al. NODDI: practical in vivo neurite orientation dispersion and density imaging of the human brain. Neuroimage 2012;61:1000-16 CrossRef Medline

29. Tournier JD, Smith R, Raffelt D, et al. MRtrix3: a fast, flexible and open software framework for medical image processing and visualisation. Neuroimage 2019;202:116137 CrossRef Medline

30. Smith RE, Tournier JD, Calamante F, et al. Anatomically-constrained tractography: improved diffusion MRI streamlines tractography through effective use of anatomical information. Neuroimage 2012;62:1924-38 CrossRef Medline

31. Smith RE, Tournier JD, Calamante F, et al. SIFT: spherical-deconvolution informed filtering of tractograms. Neuroimage 2013;67:298312 CrossRef Medline

32. Granberg T, Fan Q, Treaba CA, et al. In vivo characterization of cortical and white matter neuroaxonal pathology in early multiple sclerosis. Brain 2017;140:2912-26 CrossRef Medline

33. Hagiwara A, Kamagata K, Shimoji K, et al. White matter abnormalities in multiple sclerosis evaluated by quantitative synthetic MRI, diffusion tensor imaging, and neurite orientation dispersion and density imaging. AJNR Am J Neuroradiol 2019;40:1642-48 CrossRef Medline

34. De Santis S, Granberg T, Ouellette R, et al. Evidence of early microstructural white matter abnormalities in multiple sclerosis from multi-shell diffusion MRI. Neuroimage Clin 2019;22:101699 CrossRef Medline

35. Aung WY, Mar S, Benzinger TL. Diffusion tensor MRI as a biomarker in axonal and myelin damage. Imaging Med 2013;5:427-40 CrossRef Medline

36. Nave KA. Myelination and support of axonal integrity by glia. Nature 2010;468:244-52 CrossRef Medline

37. Trapp BD, Nave KA. Multiple sclerosis: an immune or neurodegenerative disorder? Annu Rev Neurosci 2008;31:247-69 CrossRef Medline

38. Yoshino M, Saito K, Kawasaki K, et al. The origin and development of subcortical U-fibers in gyrencephalic ferrets. Mol Brain 2020;13:37 CrossRef Medline

39. Grussu F, Schneider T, Tur C, et al. Neurite dispersion: a new marker of multiple sclerosis spinal cord pathology? Ann Clin Transl Neurol 2017;4:663-79 CrossRef Medline

40. Miki Y, Grossman RI, Udupa JK, et al. Isolated U-fiber involvement in MS: preliminary observations. Neurology 1998;50:1301-06 CrossRef Medline

41. Peterson JW, Bo L, Mork S, et al. Transected neurites, apoptotic neurons, and reduced inflammation in cortical multiple sclerosis lesions. Ann Neurol 2001;50:389-400 CrossRef Medline

42. Mainero $\mathrm{C}$, Benner $\mathrm{T}$, Radding $\mathrm{A}$, et al. In vivo imaging of cortical pathology in multiple sclerosis using ultra-high field MRI. Neurology 2009;73:941-48 CrossRef Medline

43. Beck ES, Sati P, Sethi V, et al. Improved visualization of cortical lesions in multiple sclerosis using 7T MP2RAGE. AJNR Am J Neuroradiol 2018;39:459-66 CrossRef Medline 